Non-local low-energy effective action for gravity with torsion

This article has been downloaded from IOPscience. Please scroll down to see the full text article.

1999 Class. Quantum Grav. 164057

(http://iopscience.iop.org/0264-9381/16/12/321)

View the table of contents for this issue, or go to the journal homepage for more

Download details:

IP Address: 147.96.14.16

The article was downloaded on 08/02/2013 at $17: 52$

Please note that terms and conditions apply. 


\title{
Non-local low-energy effective action for gravity with torsion
}

\author{
Antonio Dobado $†$ and Antonio L Maroto $\ddagger$ \\ $\dagger$ Departamento de Física Teórica, Universidad Complutense de Madrid, 28040 Madrid, Spain \\ ‡ Astronomy Centre, University of Sussex, Falmer, Brighton BN1 9QJ, UK
}

Received 5 May 1999

\begin{abstract}
In this work we calculate the low-energy effective action for gravity with torsion, obtained after the integration of scalar and fermionic matter fields, using the local momentum representation based on the Riemann normal coordinates expansion. By considering this expansion around different spacetime points, we also compute the non-local terms together with the more usual divergent ones. Finally, we discuss the applicability of our results to the calculation of particle production probabilities.
\end{abstract}

PACS numbers: 0462, 0450

\section{Introduction}

The absence of a quantum theory of gravitation is probably one of the most important open problems in theoretical physics. In fact, all of our knowledge about gravitation refers only to its classical aspects which are well described by general relativity and other generalizations of this theory with the same low-energy limit. On the other hand, the other interactions (strong and electroweak) are well described, even at the quantum level, by the standard model, as has been confirmed in detail over the last few years at the large electron-positron collider (LEP) and many other experiments. Moreover, it seems reasonable to think that there may exist some physical regime where the gravitational field could be treated classically, whereas matter is quantized. In fact this is the only regime that can be studied now without making an extra hypothesis beyond what has already been checked experimentally. In this very conservative approach one considers, on the one hand, the quantum gauge and matter fields propagating on a curved spacetime and, on the other hand, the dynamics of the classical degrees of freedom associated with the spacetime. This is the so-called semiclassical approximation. It is clear that spacetime curvature or torsion (see $[1,2]$ for a review) can affect quantum fields in different ways. In addition, spacetime is also affected by the presence of the quantum matter fields, as happens, for instance, in the electromagnetic field case, where fermion loops modify the electromagnetic dynamics by means of the vacuum polarization and other effects.

In order to study these new effects due to matter field loops, it is especially appropriate to use the gravitational effective action (EA). This EA is obtained after integrating out the matter fields. In general, the EA will be a non-local and non-analytical functional of the metric and the connection. The exact expression for the EA, obtained after integrating out the matter fields $W\left[g_{\mu \nu}\right]$ is not known for arbitrary spacetime geometries. However, there are several techniques that have been proposed for its approximate calculation, namely perturbation theory, in which the metric tensor is divided into a flat metric and a small fluctuation. The main difficulty of this method is that general covariance is explicitly lost [3-5]. The Schwinger-DeWitt proper time 
representation $[6,7]$ allows us to obtain a covariant asymptotic expansion for the EA but, since this expansion is local, it does not allow one to describe particle creation processes. These difficulties are intended to be solved by means of the partial resummation of the SchwingerDeWitt series [8]. There are also perturbative methods that respect general covariance, such as the so-called covariant perturbation theory [9]. Finally, the local momentum representation [10] is based on the Riemann normal coordinate expansion. This technique has the advantage of combining the usual flat spacetime methods with formally covariant expressions. It has allowed us to calculate the divergent local parts of the one-loop effective action for scalar and fermionic theories. The main aim of the present work is to find a representation of the non-local finite parts of the effective action in this formalism.

Once we know the EA, we have all the information concerning the semiclassical gravitational evolution. The corresponding equations of motion will modify the Einstein field equations taking into account quantum effects. Moreover, the EA could have a non-vanishing imaginary part, which can be interpreted as the particle production probability $[6,11]$.

In this work we show our results concerning the computation of the one-loop EA after integrating out scalar and fermionic fields by using the local momentum representation. Our computation includes not only the divergences but also the non-local finite terms that can lead to instabilities of the classical solutions by particle emission. As we will show, the use of normal coordinates allows us to obtain a useful representation of the non-local form factors. Such a representation has been successfully applied in a recent work to obtain particle production in different cosmological contexts in a remarkably easy way [11]. In addition, the results will shed some light about the boundary conditions on the metric tensor which permit a definition of the effective action.

This paper is organized as follows. In section 2 we give a brief review of the EulerHeisenberg effective Lagrangian for quantum electrodynamics (QED). This model will be a guide for the calculation of the corresponding gravitational EA. In section 3 we present the method to generate the derivative expansions of the EA by means of Riemann normal coordinates. Applying such a method to the scalar theory in the presence of gravitation, we obtain the divergences as well as the finite non-local pieces of the EA up to quadratic terms in the curvature. In section 4 we do the same with fermionic matter fields, obtaining the corresponding gravitational EA up to quadratic terms in the curvature and, in this case, also in the torsion. In particular, we apply our results to the standard model particle content. Finally, section 5 contains the main conclusions of this work and a brief discussion on their possible applications to the computation of particle production probabilities. We have also included an appendix containing the dimensional regularization formulae and some normal coordinates expansions used in the text.

\section{The Euler-Heisenberg Lagrangian}

The historical origin of the semiclassical EA can be traced back to the Euler-Heisenberg Lagrangian for QED [12]. When the momentum $p$ of photons is much smaller than the electron mass $M$, the one-loop effects, such as vacuum polarization, can be taken into account by adding local nonlinear terms to the classical electromagnetic Lagrangian. Consider the QED EA given by

$$
\begin{aligned}
\mathrm{e}^{\mathrm{i} W[A]} & =\int[\mathrm{d} \psi][\mathrm{d} \bar{\psi}] \mathrm{e}^{-\frac{1}{4} \mathrm{i} \int \mathrm{d}^{4} x F_{\mu \nu} F^{\mu \nu}} \exp \left(\mathrm{i} \int \mathrm{d}^{4} x \bar{\psi}(\mathrm{i} \not D-M+\mathrm{i} \epsilon) \psi\right) \\
& =\mathrm{e}^{-\frac{1}{4} \mathrm{i} \int \mathrm{d}^{4} x F_{\mu \nu} F^{\mu v}} \operatorname{det}(\mathrm{i} \not D-M+\mathrm{i} \epsilon)
\end{aligned}
$$


where as usual $\not D=\gamma^{\mu}\left(\partial_{\mu}-\mathrm{i} e A_{\mu}\right)$. Using dimensional regularization (see the appendix), it is possible to find the following expression up to quadratic terms in the photon field:

$$
\begin{aligned}
W[A]= & -\frac{1}{4} \int \mathrm{d}^{4} x F_{\mu \nu} F^{\mu \nu}-\mathrm{i} \operatorname{Tr} \log ((\mathrm{i} \not D-M+\mathrm{i} \epsilon)) \\
= & -\frac{1}{4} \int \mathrm{d}^{4} x F_{\mu \nu} F^{\mu \nu}+\mathrm{i} \sum_{k=1}^{\infty} \frac{(-e)^{k}}{k} \operatorname{Tr}\left[(\mathrm{i} \not \partial-M)^{-1} \not\right]^{k} \\
= & \int \mathrm{d}^{4} x\left[-\frac{1}{4} F^{\mu \nu} F_{\mu \nu}-\frac{e^{2} \Delta}{3(4 \pi)^{2}} F^{\mu \nu} F_{\mu \nu}\right. \\
& \left.+\frac{2 e^{2}}{(4 \pi)^{2}} F^{\mu \nu}\left(\frac{2}{3} \frac{M^{2}}{\square}+\frac{1}{6}\left(1-2 \frac{M^{2}}{\square}\right) F\left(-\square ; M^{2}\right)\right) F_{\mu \nu}\right]+\mathcal{O}\left(A^{4}\right)
\end{aligned}
$$

where $\Delta=N_{\epsilon}-\log \left(M^{2} / \mu^{2}\right)$, with $N_{\epsilon}=2 / \epsilon+\log 4 \pi-\gamma$ and $\gamma \simeq 0.577$ is the Euler constant. We have performed the formal Taylor expansion of the logarithm and used the expression

$$
F\left(-\square ; M^{2}\right) F_{\mu \nu}(x)=\int \mathrm{d}^{4} y \frac{\mathrm{d}^{4} p}{(2 \pi)^{4}} \mathrm{e}^{\mathrm{i} p(x-y)} F\left(p^{2} ; M^{2}\right) F_{\mu \nu}(y)
$$

with

$$
F\left(p^{2} ; M^{2}\right)=2+\int_{0}^{1} \mathrm{~d} t \log \left(1-\frac{p^{2}}{M^{2}} t(1-t)\right) .
$$

In a similar way, the inverse operator $1 / \square$ can be defined with the usual boundary conditions on the fields as

$$
\frac{1}{-\square} F_{\mu \nu}(x)=\int \mathrm{d}^{4} y \frac{\mathrm{d}^{4} p}{(2 \pi)^{4}} \mathrm{e}^{\mathrm{i} p(x-y)} \frac{1}{p^{2}+\mathrm{i} \epsilon} F_{\mu \nu}(y) .
$$

The expression (2) for the EA is non-local and has a regular massless limit. In fact, for small $p$ compared with $M$, the Mandelstam function $F\left(p^{2} ; M^{2}\right)$ behaves as

$$
F\left(p^{2} ; M^{2}\right)=-\log \left(\frac{M^{2}}{-p^{2}-\mathrm{i} \epsilon}\right)+\mathcal{O}\left(M^{2}\right) .
$$

From (2) we can see that the only contributions in the massless limit are those coming, on the one hand, from the $\Delta$ factor and, on the other hand, from the Mandelstam function. Both logarithmic contributions are equal, up to sign, so that they cancel each other and we obtain

$$
W[A]=\int \mathrm{d}^{4} x\left(-\frac{1}{4} F^{\mu \nu} F_{\mu \nu}-\frac{e^{2}}{3(4 \pi)^{2}} F^{\mu \nu} \Gamma(\square) F_{\mu \nu}\right)+\mathcal{O}\left(A^{4}\right)
$$

where we have used the following notation:

$$
\Gamma(\square)=N_{\epsilon}-\log \left(\frac{\square}{\mu^{2}}\right)
$$

to be understood, as in the previous cases, through the corresponding Fourier transform, with the $i \epsilon$ factor as shown in (6).

The EA (2) allows us to derive in an exact fashion the photon two-point one-loop Green functions. This, in turn, allows us to obtain for example the vacuum polarization. The EA can be expanded as a power series in $p^{2} / M^{2}$, and also in $A$ to obtain the well known EulerHeisenberg local Lagrangian [12]. 


\section{Integration of matter fields in a gravitational background}

Consider a scalar field in a curved spacetime. The corresponding classical action is given by

$$
S[\phi]=-\frac{1}{2} \int \mathrm{d}^{4} x \sqrt{g} \phi\left(\square+m^{2}+\xi R\right) \phi
$$

where $\square \phi=g^{\mu \nu} \nabla_{\mu} \partial_{\nu} \phi=g^{-1 / 2} \partial_{\mu}\left(g^{\mu \nu} \sqrt{g} \partial_{\nu} \phi\right)$. The non-minimal term $\xi R$ is included so that for $m=0$ and $\xi=\frac{1}{6}$, the classical Lagrangian is invariant under local conformal transformations.

The EA for the gravitational fields that arises after integrating out real scalar matter fields is given by the following expression in Lorentzian signature:

$$
\begin{aligned}
\mathrm{e}^{\mathrm{i} W\left[g_{\mu \nu}\right]} & =\int[\mathrm{d} \phi] \mathrm{e}^{\mathrm{i} S\left[g_{\mu \nu}, \phi\right]}=\int[\mathrm{d} \phi] \exp \left[-\frac{1}{2} \mathrm{i} \int \mathrm{d}^{4} x \sqrt{g} \phi\left(\square+m^{2}+\xi R-\mathrm{i} \epsilon\right) \phi\right] \\
& =(\operatorname{det} O)^{-1 / 2}
\end{aligned}
$$

where $O_{x y}\left(m^{2}\right)=\left(-\square_{y}-m^{2}-\xi R(y)+\mathrm{i} \epsilon\right) \delta^{0}(x, y)$, with $\delta^{0}(x, y)$ being the covariant Dirac delta $\delta^{0}(x, y)=g^{-1 / 2}(x) \delta(x, y)$. Therefore, we can write

$$
W\left[g_{\mu \nu}\right]=\frac{1}{2} \mathrm{i} \log \operatorname{det} O\left(m^{2}\right)=\frac{1}{2} \mathrm{i} \operatorname{Tr} \log O\left(m^{2}\right) .
$$

Since in this expression we have only integrated the scalars out, the gravitational field is treated classically. Accordingly, this EA is analogous to the classical action but including the quantum effects due to the matter fields. In addition, equation (10) is the generating functional of the Green functions containing only scalar loops and gravitational external legs.

Let us now consider a fermion field propagating in a curved spacetime with torsion [1]. The corresponding classical action will be given by

$S=\int \mathrm{d}^{4} x \sqrt{g} \bar{\psi}(\mathrm{i} \not D-M) \psi=\int \mathrm{d}^{4} x \sqrt{g} \bar{\psi}\left[\mathrm{i} \gamma^{\mu}\left(\partial_{\mu}-\frac{1}{2} \mathrm{i} \Gamma_{\mu}^{a b} \Sigma_{a b}+\frac{1}{8} \mathrm{i} S_{\mu} \gamma_{5}\right)-M\right] \psi$

where $S_{\mu}=\epsilon_{\rho \nu \lambda \mu} T^{\rho \nu \lambda}$ is the torsion pseudotrace, $\Gamma_{\mu}^{a b}$ are the Levi-Civita spin-connection components, and $\Sigma_{a b}$ are the Lorentz group generators. The fermionic EA is given by

$\mathrm{e}^{\mathrm{i} W[e, \hat{\Gamma}, A]}=\int[\mathrm{d} \psi \mathrm{d} \bar{\psi}] \exp \left(\mathrm{i} \int \mathrm{d}^{4} x \sqrt{g} \bar{\psi}(\mathrm{i} \not D-M+\mathrm{i} \epsilon) \psi\right)=\operatorname{det}(\mathrm{i} \not D-M+\mathrm{i} \epsilon)$

where $e$ denotes the vierbein, $\hat{\Gamma}$ the full connection with torsion, and $A$ collectively denotes the possible gauge fields. Accordingly,

$$
W[e, \hat{\Gamma}, A]=-\mathrm{i} \log \operatorname{det}(\mathrm{i} \not D-M+\mathrm{i} \epsilon)=-\mathrm{i} \operatorname{Tr} \log (\mathrm{i} \not D-M+\mathrm{i} \epsilon) .
$$

In the massless limit, $M=0$, the classical fermionic Lagrangian is also conformally invariant.

Since the above model does not possess self-interactions, the one-loop calculation is exact. This does not mean that it is possible to explicitly calculate the EA for an arbitrary spacetime geometry. In cases in which there is a high degree of symmetry, as in maximally symmetric manifolds, or in the so-called conformally trivial situations, i.e. conformally flat manifolds and conformally invariant theories, it is possible to find the explicit form of the modified Einstein equations coming from the EA [13].

In order to consider more general geometries, we will use an approximation scheme similar to the one used for the Euler-Heisenberg Lagrangian. It consists of treating the curvature as a small perturbation. When we integrate massive fields out, this is equivalent to considering that the Compton wavelength corresponding to the massive particle is much smaller than the characteristic length scale of the gravitational field. In this framework, the expression for the 
EA will be an expansion in metric tensor derivatives over the particle mass. Such expansion will be generated by the normal coordinates expansions of the $O\left(\mathrm{~m}^{2}\right)$ and (i $\left.\not D-M\right)$ operators. In the massless case, or if we are interested in the high-energy regime, it is possible to obtain an alternative expansion in powers of the curvatures (Riemann and Ricci tensors and scalar curvature), generically denoted by $\mathcal{R}$.

\subsection{Riemann normal coordinates}

Let $x_{0}^{\alpha}$ be the coordinates of $P$ in a given coordinate system and consider the set of geodesics passing through $P$ that we will write as $x^{\alpha}(\tau)$. We will choose the $\tau$ parameter in such a way that $x^{\alpha}(0)=x_{0}^{\alpha}$. Each of these geodesics will be characterized by the tangent vector at $P$,

$$
\xi^{\alpha}=\left.\frac{\mathrm{d} x^{\alpha}}{\mathrm{d} \tau}\right|_{\tau=0},
$$

and each point $A$ on each geodesic by a certain value of the $\tau$ parameter. The Riemann coordinates $y^{\alpha}$ of $A$ are defined as $y^{\alpha}=\xi^{\alpha} \tau$ [14]. In a neighbourhood of $P$ where any other point $A$ can be joined to $P$ by a unique geodesic (normal neighbourhood), the correspondence between the $x^{\alpha}$ and $y^{\alpha}$ coordinates is one to one.

When torsion is present and it is completely antisymmetric, the geodesic equation agrees with that obtained using the Levi-Civita connection, and therefore some given Riemann coordinates with respect to the Levi-Civita connection will also be Riemannian with respect to the connection with torsion $[15,16]$. By means of a linear real homogeneous coordinate transformation, it is possible to write the metric tensor at $y_{0}$ in the Minkowski form $\eta_{\mu \nu}$. The new coordinates are also Riemannian and they are known as Riemann normal coordinates.

Let us consider the components of some tensor field at $y$, that we will assume to be analytic functions in a neighbourhood of the normal coordinates origin $y_{0}$. From their Taylor expansion around $y_{0}$ we can obtain an expression that is written as a series in curvatures and their covariant derivatives. In particular, for the metric tensor components we obtain [14]

$$
\begin{aligned}
g_{\mu \nu}(y)=\eta_{\mu \nu}+ & \frac{1}{3} R_{\mu \alpha \nu \beta}\left(y_{0}\right) y^{\alpha} y^{\beta}-\frac{1}{6} R_{\mu \alpha \nu \beta ; \gamma}\left(y_{0}\right) y^{\alpha} y^{\beta} y^{\gamma} \\
& +\left[\frac{1}{20} R_{\mu \alpha \nu \beta ; \gamma \delta}\left(y_{0}\right)+\frac{2}{45} R_{\alpha \mu \beta \lambda}\left(y_{0}\right) R_{\gamma \nu \delta}^{\lambda}\left(y_{0}\right)\right] y^{\alpha} y^{\beta} y^{\gamma} y^{\delta}+\mathcal{O}\left(\partial^{5}\right) .
\end{aligned}
$$

Here, $\mathcal{O}\left(\partial^{5}\right)$ denotes terms with five or more derivatives and the indices in those tensors evaluated in $y_{0}$ are raised and lowered with the flat metric $\eta_{\mu \nu}$. In the appendix, we have written the expansions corresponding to the metric determinant and other useful expressions. It is important to note that (16) is a superposition of two kinds of expansions: on one hand, terms are organized by the number of metric derivatives but, on the other hand, those terms with a certain number of derivatives can be classified according to the number of curvature tensors they have.

It will also be useful to introduce the following relation: $2 \sigma\left(x, x^{\prime}\right)=y_{\alpha} y^{\alpha}$, where the biscalar $\sigma\left(x, x^{\prime}\right)$ represents half of the geodesic distance between the $x$ and $x^{\prime}$ points and $y^{\alpha}$ denotes the normal coordinates of the $x$ point with the origin at $x^{\prime}$. However, $\partial_{\alpha}^{x} \sigma\left(x, x^{\prime}\right)$ is a tangent vector at $x$ to the geodesic joining $x$ and $x^{\prime}$, whose length equals the geodesic distance between these two points and it is oriented in the $x^{\prime} \rightarrow x$ direction. In turn, $\partial_{\alpha}^{x^{\prime}} \sigma\left(x, x^{\prime}\right)$ is tangent to the same geodesic at $x^{\prime}$, with the same modulus and oriented in the opposite sense. 
In normal coordinates with the origin at $x^{\prime}$, according to the previous expressions, we can write

$$
\sigma_{\alpha}\left(x, x^{\prime}\right)=\frac{\partial}{\partial x^{\alpha}} \sigma\left(x, x^{\prime}\right)=y_{\alpha},
$$

i.e. $y_{\alpha}$ are components of a vector tangent at the origin.

The use of normal coordinates, apart from being basic to obtaining the derivative expansions of the EA, allows us to work in momentum space in a similar way to the flat spacetime. Let us consider some scalar function $f\left(x, y_{0}\right)$ with normal coordinates with the origin at $y_{0}$. We can define its covariant Fourier transform through [10]:

$$
f\left(x, y_{0}\right)=\int \frac{\mathrm{d}^{4} k}{(2 \pi)^{4}} \hat{f}\left(k, y_{0}\right) \mathrm{e}^{-\mathrm{i} k x} .
$$

In a similar way we can introduce the covariant Dirac delta

$$
\delta^{0}\left(x, y_{0}\right)=\int \frac{\mathrm{d}^{4} k}{(2 \pi)^{4}} \mathrm{e}^{-\mathrm{i} k x} .
$$

As long as one of the delta arguments is the coordinate origin, we will have $\delta^{0}\left(x, y_{0}\right)=$ $\delta\left(x, y_{0}\right) g^{-1 / 2}\left(y_{0}\right)=\delta\left(x, y_{0}\right)$. In the general case (arbitrary arguments) there is also a covariant definition whose expression in arbitrary coordinates is given by [8]

$$
\begin{gathered}
\delta^{0}(x, y)=g^{1 / 4}(x) g^{1 / 4}(y) \Delta^{1 / 2}\left(x, y_{0}\right) \Delta^{1 / 2}\left(y, y_{0}\right) \int \frac{\mathrm{d}^{4} k}{(2 \pi)^{4}} g^{-1 / 2}\left(y_{0}\right) \\
\times \exp \left(\mathrm{i} k_{\mu}\left(\sigma^{\mu}\left(y, y_{0}\right)-\sigma^{\mu}\left(x, y_{0}\right)\right)\right)
\end{gathered}
$$

where $k_{\mu}, \sigma_{\mu}\left(y, y_{0}\right)$ and $\sigma_{\mu}\left(x, y_{0}\right)$ are tangent vectors at $y_{0}$ and $\Delta\left(x, x^{\prime}\right)$ is the Van VleckMorette determinant, defined as $\Delta\left(x, x^{\prime}\right)=g^{-1 / 2}(x) \operatorname{det}\left(-\nabla_{\mu^{\prime}} \nabla_{\nu} \sigma\left(x, x^{\prime}\right)\right) g^{-1 / 2}\left(x^{\prime}\right)$. If we take $y_{0}$ as the origin, we will have $\Delta\left(x, y_{0}\right)=g^{-1 / 2}(x)$, this expression then reduces to

$$
\delta^{0}(x, y)=g^{-1 / 2}(x) \int \frac{\mathrm{d}^{4} k}{(2 \pi)^{4}} \exp \left(-\mathrm{i} k_{\mu}\left(x^{\mu}-y^{\mu}\right)\right) .
$$

All of these definitions are valid only in normal neighbourhoods of the origin in which geodesics do not intersect.

\subsection{Derivative expansions}

In normal coordinates there is a privileged point around which we perform the expansions. In addition, the different curvature tensors are defined on the tangent plane corresponding to that point. This fact, together with the general coordinate invariance of the EA, will allow us to obtain a covariant derivative expansion for the effective Lagrangian around the origin. In the following we will discuss in detail the scalar case, although the procedure is the same for fields with different spin.

Let us start with the scalar EA (11). Using the normal coordinates expansion for the metric tensor it is easy to split the operator $O_{x y}\left(m^{2}\right)=\left(-\square_{y}-m^{2}-\xi R(y)+\mathrm{i} \epsilon\right) \delta^{0}(x, y)$ into a free part that coincides with the flat spacetime Klein-Gordon operator

$$
A_{x y}\left(m^{2}\right)=\left(-\square_{0}^{y}-m^{2}+\mathrm{i} \epsilon\right) \delta^{0}(x, y)
$$


with $\square_{0}^{y}=\eta^{\mu v} \partial_{\mu}^{y} \partial_{\nu}^{y}$, and the interaction part $B_{x y}$ that includes all of the curvature dependence:

$$
\begin{aligned}
& B_{x y}=\left[-\frac{2}{3} R_{\rho}^{\lambda}\left(y_{0}\right) y^{\rho} \partial_{\lambda}^{y}+\frac{1}{3} R^{\mu}{ }_{\epsilon}{ }^{\nu}{ }_{\beta}\left(y_{0}\right) y^{\epsilon} y^{\beta} \partial_{\mu}^{y} \partial_{\nu}^{y}-\xi R\left(y_{0}\right)-\left(\frac{1}{20} R_{\alpha}{ }^{\nu} ; \beta \gamma\left(y_{0}\right)\right.\right.
\end{aligned}
$$

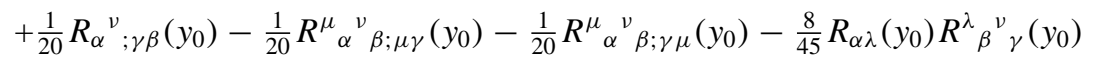

$$
\begin{aligned}
& +\frac{1}{15} R_{\alpha}{ }^{\mu}{ }_{\beta \lambda}\left(y_{0}\right) R^{\lambda}{ }_{\mu}{ }^{\nu}{ }_{\gamma}\left(y_{0}\right)+\frac{4}{45} R_{\alpha}{ }^{\mu}{ }_{\beta \lambda}\left(y_{0}\right) R^{\lambda}{ }^{\lambda}{ }^{\nu}{ }_{\mu}\left(y_{0}\right)+\frac{1}{40} R_{\alpha \beta ; \gamma}{ }^{\nu}\left(y_{0}\right)
\end{aligned}
$$

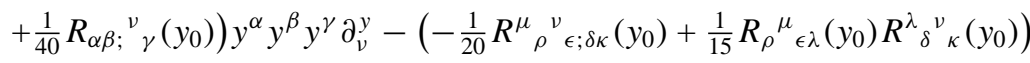

$$
\begin{aligned}
& \left.\times y^{\rho} y^{\epsilon} y^{\delta} y^{\kappa} \partial_{\mu}^{y} \partial_{v}^{y}-\xi \frac{1}{2} R_{; \alpha \beta}\left(y_{0}\right) y^{\alpha} y^{\beta}\right] \delta^{0}(x, y)+\cdots \text {. }
\end{aligned}
$$

We have only written the two- and four-derivatives contributions since terms with an odd number of metric derivatives are shown to be irrelevant for the final result. Therefore, we have $O_{x y}\left(m^{2}\right)=A_{x y}\left(m^{2}\right)+B_{x y}$

We will also assume that spacetime is asymptotically flat and this will allows us to discard total derivatives of the curvatures in the EA. We have included the covariant Dirac delta function $\delta^{0}(x, y)$ in the definition of the free operator so that we can use the covariant integration measure $\mathrm{d}^{4} x g^{1 / 2}(x)$. Taking all this into account we can write the EA as

$$
W\left[g_{\mu \nu}\right]=\int \mathrm{d}^{4} x \mathcal{L}_{e f f}(x)=\frac{1}{2} \mathrm{i} \operatorname{Tr} \log O\left(m^{2}\right)=\frac{1}{2} \mathrm{i} \operatorname{Tr} \log (A+B) .
$$

As long as the calculations are done in normal coordinates with respect to the $y_{0}$ point, any term in the effective Lagrangian is evaluated in that point. For that reason the integration involved in the Tr symbol cannot be done immediately. This, in turn, keeps us from expanding the logarithm, which only makes sense inside the trace. A way to avoid the problem consists of formally differentiating the EA with respect to $m^{2}$ [17]:

$$
\begin{aligned}
\frac{\mathrm{d}}{\mathrm{d} m^{2}} W\left[g_{\mu \nu}\right] & =-\frac{\mathrm{i}}{2} \operatorname{Tr} \frac{1}{O\left(m^{2}\right)}=-\frac{\mathrm{i}}{2} \operatorname{Tr}\left((A+B)^{-1}\right) \\
& =-\frac{\mathrm{i}}{2} \operatorname{Tr}\left(A^{-1}-A^{-1} B A^{-1}+A^{-1} B A^{-1} B A^{-1}-\cdots\right) .
\end{aligned}
$$

In the last step the inverse of $A+B$ has been expanded. This can be done without taking the functional trace.

The lowest-order term in $B$ is linear in the Riemann tensor and therefore it contains two metric derivatives. The term in (25) with two $B$ factors will be $\mathcal{O}\left(\mathcal{R}^{2}\right)$ and will contain at least 4-metric derivatives, etc. Since we are only interested in the result up to $\mathcal{O}\left(\mathcal{R}^{2}\right)$, it will be enough to consider the first three terms in (25).

\subsection{Divergent parts}

In order to calculate the divergent parts of the EA, we consider the previous expansions. Since normal coordinates allow us to perform momentum space integrations, we can use the flat spacetime regularization techniques such as dimensional regularization. Our calculation method is based on the local momentum representation proposed in [10], but adapted to the EA techniques. The results agree with the well known expressions in [13].

First, we find the scalar propagator $A_{x y}^{-1}$, given by $A_{x y} A_{y z}^{-1}=\delta^{0}(x, z)$, where we have used the DeWitt generalized summation convention for repeated indices. Using (21), we can write this equation as

$$
\int \mathrm{d}^{4} y g^{1 / 2}(y)\left(-\square_{0}^{y}-m^{2}+\mathrm{i} \epsilon\right) \frac{\delta(x, y)}{g^{1 / 2}(x)} g^{-1 / 2}(y) \int \frac{\mathrm{d}^{4} k}{(2 \pi)^{4}} \mathrm{e}^{-\mathrm{i} k(y-z)} \tilde{G}(k)=\frac{\delta(x, z)}{g^{1 / 2}(x)} .
$$


The $G(k)$ function is easily obtained and finally the propagator reads

$$
A_{y z}^{-1}=\frac{1}{g^{1 / 2}(y)} \int \frac{\mathrm{d}^{4} k}{(2 \pi)^{4}} \mathrm{e}^{-\mathrm{i} k(y-z)} \frac{1}{k^{2}-m^{2}+\mathrm{i} \epsilon} .
$$

The first term in the EA expansion (25) can be immediately evaluated and it reads

$$
A_{y_{0} y_{0}}^{-1}=\int \mathrm{d} \tilde{k} \frac{1}{k^{2}-m^{2}}=-\frac{\mathrm{i}}{(4 \pi)^{D / 2}} \frac{\Gamma(1-D / 2) \mu^{\epsilon}}{\left(m^{2}\right)^{1-D / 2}}
$$

with the notation $\mathrm{d} \tilde{k}=\mathrm{d}^{D} k \mu^{\epsilon} /(2 \pi)^{D}$. When $y_{0}$ appears as a repeated subindex it must be understood that the integration in $y_{0}$ has not been done. This final integration, which corresponds to the trace in (25), will be performed below in an explicit way. In the last step we have used equation (A1) from the appendix. Performing the $m^{2}$ integration we have the lowest-order term in the effective Lagrangian:

$$
\mathcal{L}_{\text {div }}^{(0)}\left(y_{0}\right)=\frac{1}{64 \pi^{2}} m^{4}\left(\Delta+\frac{3}{2}\right)
$$

where we have defined $\Delta=N_{\epsilon}+\log \left(\mu^{2} / m^{2}\right)$. As is well known, this first term will give rise to the cosmological constant renormalization. The integration constant can be set to zero without losing generality since it can be absorbed in the renormalization procedure as we will show below.

Up to two metric derivatives, only the second term in (25) contributes:

$$
\begin{aligned}
\left(A_{y_{0} y}^{-1} B_{y z} A_{z y_{0}}^{-1}\right)^{(2)} & =\int \mathrm{d}^{4} y g^{1 / 2}(y) \mathrm{d}^{4} z g^{1 / 2}(z) \int \mathrm{d} \tilde{k} \frac{\mathrm{e}^{\mathrm{i} k y}}{k^{2}-m^{2}}\left(-\frac{2}{3} R_{\rho}^{\lambda}\left(y_{0}\right) z^{\rho} \partial_{\lambda}^{z}\right. \\
+ & \left.\frac{1}{3} R^{\mu} \epsilon^{\nu}{ }_{\beta}\left(y_{0}\right) z^{\epsilon} z^{\beta} \partial_{\mu}^{z} \partial_{\nu}^{z}-\xi R\left(y_{0}\right)\right) \frac{\delta(y, z)}{g^{1 / 2}(y)} g^{-1 / 2}(z) \int \mathrm{d} \tilde{q} \frac{\mathrm{e}^{-\mathrm{i} q z}}{q^{2}-m^{2}} .
\end{aligned}
$$

Integrating by parts and removing the coordinates $z$ through $z \rightarrow \mathrm{i} \partial_{q}$, we can rewrite this expression as

$$
\begin{aligned}
\left(A_{y_{0} y}^{-1} B_{y z} A_{z y_{0}}^{-1}\right)^{(2)} & =\int \mathrm{d}^{4} z \mathrm{~d} \tilde{p} \mathrm{~d} \tilde{q} \frac{\mathrm{e}^{-\mathrm{i} p z}}{q^{2}-m^{2}}\left(\frac{\frac{1}{3} R\left(y_{0}\right)-\xi R\left(y_{0}\right)}{(q-p)^{2}-m^{2}}-\frac{2}{3} \frac{R^{\mu \nu}\left(y_{0}\right) q_{\mu} q_{\nu}}{\left((q-p)^{2}-m^{2}\right)^{2}}\right) \\
& =\frac{\mathrm{i}}{(4 \pi)^{D / 2}} \frac{\Gamma(2-D / 2)}{\left(m^{2}\right)^{2-D / 2}}\left(\frac{1}{6}-\xi\right) R\left(y_{0}\right) .
\end{aligned}
$$

In the last step we have done the $z$ integration first and then those corresponding to the momenta. Finally, integrating in $m^{2}$ we find the effective Lagrangian contribution to order $\mathcal{O}\left(\partial^{2}\right)$ :

$$
\mathcal{L}_{\text {div }}^{(2)}\left(y_{0}\right)=-\frac{1}{32 \pi^{2}} m^{2}(\Delta+1)\left(\frac{1}{6}-\xi\right) R\left(y_{0}\right) .
$$

Now we derive the divergences with 4-metric derivatives. In this case, there are contributions from $A^{-1} B A^{-1}$ and $A^{-1} B A^{-1} B A^{-1}$ :

$$
\begin{aligned}
& \left(A_{y_{0} y}^{-1} B_{y z} A_{z y_{0}}^{-1}\right)^{(4)}=\int \mathrm{d}^{4} y g^{1 / 2}(y) \mathrm{d}^{4} z g^{1 / 2}(z) \\
& \times \int \mathrm{d} \tilde{k} \frac{\mathrm{e}^{\mathrm{i} k y}}{k^{2}-m^{2}}\left[-\left(\frac{1}{20} R_{\alpha}{ }^{\nu} ; \beta \gamma\left(y_{0}\right)+\frac{1}{20} R_{\alpha}{ }^{\nu} ; \gamma \beta\left(y_{0}\right)-\frac{1}{20} R_{\alpha}^{\mu}{ }^{\nu}{ }_{\beta ; \mu \gamma}\left(y_{0}\right)\right.\right. \\
& -\frac{1}{20} R_{\alpha \beta ; \gamma \mu}^{\mu}\left(y_{0}\right)-\frac{8}{45} R_{\alpha \lambda}\left(y_{0}\right) R^{\lambda}{ }^{\nu}{ }^{\nu}{ }_{\gamma}\left(y_{0}\right)+\frac{1}{15} R_{\alpha}{ }^{\mu}{ }_{\beta \lambda}\left(y_{0}\right) R_{\mu}^{\lambda}{ }^{\nu}{ }_{\gamma}\left(y_{0}\right) \\
& \left.+\frac{4}{45} R_{\alpha}{ }_{\beta \lambda}{ }_{\beta \lambda}\left(y_{0}\right) R^{\lambda} \gamma^{\nu}{ }_{\mu}\left(y_{0}\right)+\frac{1}{40} R_{\alpha \beta ; \gamma}{ }^{\nu}\left(y_{0}\right)+\frac{1}{40} R_{\alpha \beta ;}{ }^{\nu}{ }^{\nu}\left(y_{0}\right)\right) z^{\alpha} z^{\beta} z^{\gamma} \partial_{v}^{z} \\
& +\left(\frac{1}{20} R_{\rho}^{\mu}{ }_{\rho}^{v}{ }_{\epsilon ; \delta \kappa}\left(y_{0}\right)-\frac{1}{15} R_{\rho}{ }^{\mu}{ }_{\epsilon \lambda}\left(y_{0}\right) R_{\delta}^{\lambda{ }^{v}{ }_{\kappa}}\left(y_{0}\right)\right) z^{\rho} z^{\epsilon} z^{\delta} z^{\kappa} \partial_{\mu}^{z} \partial_{v}^{z} \\
& \left.-\xi \frac{1}{2} R_{; \alpha \beta}\left(y_{0}\right) z^{\alpha} z^{\beta}\right] \frac{\delta(y, z)}{g^{1 / 2}(y)} g^{-1 / 2}(z) \int \mathrm{d} \tilde{q} \frac{\mathrm{e}^{-\mathrm{i} q z}}{q^{2}-m^{2}} .
\end{aligned}
$$


Removing the coordinates as before and integrating in positions and momenta we find

$$
\begin{gathered}
\left(A_{y_{0} y}^{-1} B_{y z} A_{z y_{0}}^{-1}\right)^{(4)}=-\frac{\mathrm{i}}{(4 \pi)^{D / 2}} \frac{\Gamma(3-D / 2)}{\left(m^{2}\right)^{3-D / 2}}\left(-\frac{1}{180} R^{\mu \nu \lambda \rho}\left(y_{0}\right) R_{\mu \nu \lambda \rho}\left(y_{0}\right)\right. \\
\left.-\frac{1}{270} R^{\mu \nu}\left(y_{0}\right) R_{\mu \nu}\left(y_{0}\right)+\frac{1}{6}\left(\frac{1}{5}-\xi\right) \square R\left(y_{0}\right)\right) .
\end{gathered}
$$

Notice that this result is finite. The divergences will appear when performing the $m^{2}$ integration. We add to this term the lowest-order contribution from the next one, namely

$$
\begin{aligned}
\left(A_{y_{0} y}^{-1} B_{y z} A_{z t}^{-1} B_{t u} A_{u y_{0}}^{-1}\right)^{(4)}=\int \mathrm{d}^{4} y g^{1 / 2}(y) \mathrm{d}^{4} z g^{1 / 2}(z) \mathrm{d}^{4} t g^{1 / 2}(t) \mathrm{d}^{4} u g^{1 / 2}(u) \\
\quad \times \int \mathrm{d} \tilde{k} \mathrm{~d} \tilde{q} \mathrm{~d} \tilde{p} \frac{\mathrm{e}^{\mathrm{i} k y}}{k^{2}-m^{2}}\left(-\frac{2}{3} R_{\rho}^{\lambda}\left(y_{0}\right) z^{\rho} \partial_{\lambda}^{z}+\frac{1}{3} R^{\mu} \epsilon^{v}{ }_{\beta}\left(y_{0}\right) z^{\epsilon} z^{\beta} \partial_{\mu}^{z} \partial_{v}^{z}-\xi R\left(y_{0}\right)\right) \\
\quad \times \frac{\delta(y, z)}{g^{1 / 2}(y)} g^{-1 / 2}(z) \frac{\mathrm{e}^{-\mathrm{i} q(z-t)}}{q^{2}-m^{2}}\left(-\frac{2}{3} R_{\rho}^{\lambda}\left(y_{0}\right) u^{\rho} \partial_{\lambda}^{u}+\frac{1}{3} R^{\mu}{ }_{\epsilon}{ }^{v}{ }_{\beta}\left(y_{0}\right) u^{\epsilon} u^{\beta} \partial_{\mu}^{u} \partial_{v}^{u}\right. \\
\left.-\xi R\left(y_{0}\right)\right) \frac{\delta(t, u)}{g^{1 / 2}(t)} g^{-1 / 2}(u) \frac{\mathrm{e}^{-i p u}}{p^{2}-m^{2}} .
\end{aligned}
$$

In a similar fashion to the previous case we find the results for the regularized integrals:

$$
\begin{aligned}
& \left(A_{y_{0} y}^{-1} B_{y z} A_{z t}^{-1} B_{t u} A_{u y_{0}}^{-1}\right)^{(4)} \\
& \quad=-\frac{\mathrm{i}}{(4 \pi)^{D / 2}} \frac{\Gamma(3-D / 2)}{\left(m^{2}\right)^{3-D / 2}}\left(\frac{1}{2}\left(\frac{1}{6}-\xi\right)^{2} R\left(y_{0}\right)^{2}-\frac{1}{108} R^{\mu \nu}\left(y_{0}\right) R_{\mu \nu}\left(y_{0}\right)\right) .
\end{aligned}
$$

Subtracting (34) from (36) and integrating in $\mathrm{m}^{2}$ we obtain the divergent Lagrangian up to $\mathcal{O}\left(\partial^{4}\right)$ :

$$
\begin{gathered}
\mathcal{L}_{\text {div }}^{(4)}\left(y_{0}\right)=\frac{\Delta}{32 \pi^{2}}\left(\frac{1}{180} R^{\mu \nu \lambda \rho}\left(y_{0}\right) R_{\mu \nu \lambda \rho}\left(y_{0}\right)-\frac{1}{180} R^{\mu \nu}\left(y_{0}\right) R_{\mu \nu}\left(y_{0}\right)\right. \\
\left.-\frac{1}{6}\left(\frac{1}{5}-\xi\right) \square R\left(y_{0}\right)+\frac{1}{2}\left(\frac{1}{6}-\xi\right)^{2} R\left(y_{0}\right)^{2}\right) .
\end{gathered}
$$

Comparing with the well known Schwinger-DeWitt expansion, we see that $\mathcal{L}_{\text {div }}^{(2)}\left(y_{0}\right)$ is proportional to $a_{1}\left(O, y_{0}\right)$ and $\mathcal{L}_{\text {div }}^{(4)}\left(y_{0}\right)$ to $a_{2}\left(O, y_{0}\right)$. As long as the above expressions are scalars they will have the same form in any coordinate system, not necessarily geodesic. We can then perform a coordinate change and integrate to obtain the corresponding EA:

$$
W\left[g_{\mu \nu}\right]_{d i v}=\int \mathrm{d}^{4} x \mathcal{L}_{d i v}(x) .
$$

We have included in $\mathcal{L}_{\text {div }}(x)$ the $g^{1 / 2}(x)$ factor coming from the integration measure. It is possible, in principle, that when doing the coordinate change, new non-covariant terms appear provided they vanish only for geodesic coordinates. However, in the absence of gravitational anomalies the EA is a scalar and accordingly such terms are not permitted. On the other hand, terms with an odd number of derivatives yield terms with an odd number of momenta in the numerator which vanish in dimensional regularization. The above are the only possible divergences, higher-derivative terms give rise to momentum integrals with more momenta in the denominator that turn out to be finite.

Apart from the divergences, which are purely local contributions, the EA also contains finite non-local pieces that are responsible for the pair-creation processes. We have seen that in the QED case (2), the massless limit is well defined due to the cancellation between the mass logarithmic dependence associated with the divergences and that coming from the non-local 
pieces. This fact allows us to extract some information about the non-local terms from the knowledge of the divergences. In the next section we will use this connection to find part of the non-local structure of the gravitational EA by means of a point-splitting procedure in the divergences.

\subsection{Non-local contributions}

In the previous section we have obtained local contributions up to order $\mathcal{O}\left(\mathcal{R}^{2}\right)$. If we continue the calculation to higher orders, we would obtain a power series with terms of the following type:

$$
\frac{\nabla^{n} \mathcal{R}^{p}\left(y_{0}\right)}{m^{2 p+n-4}}
$$

where $\nabla$ denotes the covariant derivative. This is a typical derivative (or adiabatic) expansion which is only valid at low energies.

All the terms in (39) can be classified by the number of curvatures they contain. Those terms with a fixed number $n$ of curvatures will give rise to a series with an increasing number of covariant derivatives. If we could add all of these terms together, we would obtain non-local contributions that would provide the exact $n$-point Green functions (with curvatures in the external legs) $[8,9,18]$. These Green functions will be valid for any value of the mass $m$, as in the QED case (2).

In this section we propose a method that effectively carries out that resummation for the quadratic operators, i.e we are interested in those terms of the form $\nabla^{n} \mathcal{R}^{2}$. The basic idea is to perform a point-splitting in the quadratic parts in curvatures in (33) and (35); at the end of the section we will argue that this procedure gives rise to the correct resummation up to $\mathcal{O}\left(m^{2} \mathcal{R}^{2}\right)$ terms.

As we have just commented, all the terms in (39) are local and finite. The reason why we have not obtained non-local terms as in (2) is that the normal coordinate expansions are performed around a single point $y_{0}$. In (33) and (35) there are products of curvatures evaluated at the same point, i.e. $\mathcal{R}\left(y_{0}\right) \mathcal{R}\left(y_{0}\right)$. Using again the normal coordinates expansion we can rewrite these products as

$$
\begin{aligned}
\mathcal{R}\left(y_{0}\right) \mathcal{R}\left(y_{0}\right)= & \mathcal{R}\left(y_{0}\right) \mathcal{R}(y)+\left(\mathcal{R}\left(y_{0}\right) \nabla \mathcal{R}\left(y_{0}\right) y+\mathcal{R}\left(y_{0}\right) \nabla^{2} \mathcal{R}\left(y_{0}\right) y y+\cdots\right) \\
& +\left(\mathcal{R}\left(y_{0}\right) \mathcal{R}\left(y_{0}\right) \mathcal{R}\left(y_{0}\right) y y+\mathcal{R}\left(y_{0}\right) \nabla \mathcal{R}\left(y_{0}\right) \mathcal{R}\left(y_{0}\right) y y y+\cdots\right)+\cdots .
\end{aligned}
$$

This expression allows us to split the points, the price we pay is the modification of the coefficients of the infinite higher-order terms. We will not modify the linear part in $\mathcal{R}$ in (33). Let us first obtain the non-local result and then we will argue that the new terms $\nabla^{n} \mathcal{R}^{2}$ generated in (40) will exactly cancel those coming from the expansion in (25).

By means of the above point-splitting, the $\mathcal{R}^{2}$ contributions in (33) and (35) will remain as

$$
\begin{aligned}
\left(A_{y_{0} y}^{-1} B_{y z} A_{z y_{0}}^{-1}\right)^{\left(\mathcal{R}^{2}\right)} & =\int \mathrm{d}^{4} y g^{1 / 2}(y) \mathrm{d}^{4} z g^{1 / 2}(z) \int \mathrm{d} \tilde{k} \frac{\mathrm{e}^{\mathrm{i} k y}}{k^{2}-m^{2}}\left[\left(\frac{8}{45} R_{\alpha \lambda}\left(y_{0}\right) R^{\lambda}{ }_{\beta}{ }^{\nu}{ }_{\gamma}(y)\right.\right. \\
& \left.-\frac{1}{15} R_{\alpha}{ }^{\mu}{ }_{\beta \lambda}\left(y_{0}\right) R^{\lambda}{ }_{\mu}{ }^{\nu}{ }_{\gamma}(y)-\frac{4}{45} R_{\alpha}{ }^{\mu}{ }_{\beta \lambda}\left(y_{0}\right) R^{\lambda}{ }_{\gamma}{ }^{\nu}{ }_{\mu}(y)\right) z^{\alpha} z^{\beta} z^{\gamma} \partial_{\nu}^{z} \\
& \left.-\frac{1}{15} R_{\rho}{ }^{\mu} \epsilon \lambda\left(y_{0}\right) R^{\lambda}{ }^{{ }^{\nu}}{ }^{\nu}(y) z^{\rho} z^{\epsilon} z^{\delta} z^{k} \partial_{\mu}^{z} \partial_{\nu}^{z}\right] \frac{\delta(y, z)}{g^{1 / 2}(y)} g^{-1 / 2}(z) \\
& \times \int \mathrm{d} \tilde{q} \frac{\mathrm{e}^{-\mathrm{i} q z}}{q^{2}-m^{2}}+\mathcal{O}\left(\nabla^{2} \mathcal{R}^{2}\right)
\end{aligned}
$$


and

$$
\begin{aligned}
\left(A_{y_{0} y}^{-1} B_{y z} A_{z t}^{-1}\right. & \left.B_{t u} A_{u y_{0}}^{-1}\right)^{\left(\mathcal{R}^{2}\right)}=\int \mathrm{d}^{4} y g^{1 / 2}(y) \mathrm{d}^{4} z g^{1 / 2}(z) \mathrm{d}^{4} t g^{1 / 2}(t) \mathrm{d}^{4} u g^{1 / 2}(u) \\
& \times \int \mathrm{d} \tilde{k} \mathrm{~d} \tilde{q} \mathrm{~d} \tilde{p} \frac{\mathrm{e}^{\mathrm{i} k y}}{k^{2}-m^{2}} \frac{\mathrm{e}^{-\mathrm{i} p u}}{p^{2}-m^{2}} \\
& \times\left(-\frac{2}{3} R_{\rho}^{\lambda}\left(y_{0}\right) z^{\rho} \partial_{\lambda}^{z}+\frac{1}{3} R^{\mu}{ }_{\epsilon}{ }^{\nu}\left(y_{0}\right) z^{\epsilon} z^{\beta} \partial_{\mu}^{z} \partial_{v}^{z}-\xi R\left(y_{0}\right)\right) \\
& \times \frac{\delta(y, z)}{g^{1 / 2}(y)} g^{-1 / 2}(z) \frac{\mathrm{e}^{-\mathrm{i} q(z-t)}}{q^{2}-m^{2}} \\
& \times\left(-\frac{2}{3} R_{\rho}^{\lambda}(t) u^{\rho} \partial_{\lambda}^{u}+\frac{1}{3} R^{\mu} \epsilon^{\nu}{ }_{\beta}(t) u^{\epsilon} u^{\beta} \partial_{\mu}^{u} \partial_{v}^{u}-\xi R(t)\right) \frac{\delta(t, u)}{g^{1 / 2}(t)} g^{-1 / 2}(u) \\
& +\mathcal{O}\left(\nabla^{2} \mathcal{R}^{2}\right)
\end{aligned}
$$

where, as mentioned before, $\mathcal{O}\left(\nabla^{2} \mathcal{R}^{2}\right)$ denotes local finite terms with two curvatures and an arbitrary even number of derivatives. There are in principle different ways of performing the splitting, depending on the choice of the pair of points, but all them are equivalent up to higher-order terms as can be seen from (40).

Removing the explicit coordinates occurrences, we obtain

$$
\begin{gathered}
\left(A_{y_{0} y}^{-1} B_{y z} A_{z t}^{-1} B_{t u} A_{u y_{0}}^{-1}\right)^{\left(R^{2}\right)}=\int \mathrm{d}^{4} t \mathrm{~d} \tilde{q} \mathrm{~d} \tilde{p} \mathrm{e}^{\mathrm{i} p t}\left(\frac{\left(\frac{1}{3}-\xi\right) R\left(y_{0}\right)}{\left(q^{2}-m^{2}\right)^{2}}-\frac{2}{3} R^{\mu v}\left(y_{0}\right) \frac{q_{\mu} q_{v}}{\left(q^{2}-m^{2}\right)^{3}}\right) \\
\times\left(\frac{-\xi R(t)}{\left((p+q)^{2}-m^{2}\right)}+\frac{2}{3} \frac{R^{\mu v}(t)(p+q)_{\mu}(p+q)_{v}}{\left((p+q)^{2}-m^{2}\right)^{2}}\right)+\mathcal{O}\left(\nabla^{2} \mathcal{R}^{2}\right) .
\end{gathered}
$$

Using equations (A2)-(A4) from the appendix, neglecting higher-order terms and integrating in $m^{2}$, we obtain the following contributions to the effective Lagrangian:

$$
\begin{aligned}
\mathcal{L}_{1}^{\left(\mathcal{R}^{2}\right)}\left(y_{0}\right)= & \frac{1}{32 \pi^{2}} \int \mathrm{d}^{4} t \mathrm{~d} \tilde{p} \mathrm{e}^{\mathrm{i} p t}\left(\Delta-F\left(p^{2} ; m^{2}\right)\right) \\
& \times\left[\frac{1}{2}\left(\frac{1}{6}-\xi\right)^{2} R\left(y_{0}\right) R(t)-\frac{1}{108} R_{\mu \nu}\left(y_{0}\right) R^{\mu \nu}(t)\right]+\mathcal{O}\left(\nabla^{2} \mathcal{R}^{2}\right) .
\end{aligned}
$$

where $F\left(p^{2} ; m^{2}\right)$ is given in (4).

Apart from the local divergent and non-local finite terms, in the dimensional regularization procedure, finite local terms do arise. However, their coefficients will be absorbed in the definition of the renormalized parameters and they will not be considered explicitly.

In the same form as before we obtain from (42)

$$
\begin{aligned}
\mathcal{L}_{2}^{\left(\mathcal{R}^{2}\right)}\left(y_{0}\right)= & \frac{1}{32 \pi^{2}} \int \mathrm{d}^{4} t \mathrm{~d} \tilde{p} \mathrm{e}^{\mathrm{i} p t}\left(\Delta-F\left(p^{2} ; m^{2}\right)\right) \\
& \times\left[\frac{1}{180} R^{\mu \nu \lambda \rho}\left(y_{0}\right) R_{\mu \nu \lambda \rho}(t)+\frac{1}{270} R^{\mu \nu}\left(y_{0}\right) R_{\mu \nu}(t)\right]+\mathcal{O}\left(\nabla^{2} \mathcal{R}^{2}\right) .
\end{aligned}
$$

Adding both contributions in (44) and (45) and including the zero and two derivatives divergent contributions given in (29) and (32), we can write the non-local EA in a slightly different notation:

$$
\begin{aligned}
W\left[g_{\mu \nu}\right]=\frac{1}{32 \pi^{2}} \int \mathrm{d}^{4} x \sqrt{g}\left[\frac{1}{2} m^{4}\left(\Delta+\frac{3}{2}\right)-m^{2}(\Delta+1)\left(\frac{1}{6}-\xi\right) R(x)\right. \\
+\Delta\left(\frac{1}{180} R^{\mu \nu \lambda \rho}(x) R_{\mu \nu \lambda \rho}(x)-\frac{1}{180} R^{\mu \nu}(x) R_{\mu \nu}(x)+\frac{1}{2}\left(\frac{1}{6}-\xi\right)^{2} R(x)^{2}\right) \\
\quad-\left(\frac{1}{180} R^{\mu \nu \lambda \rho}(x) F\left(\square ; m^{2}\right) R_{\mu \nu \lambda \rho}(x)-\frac{1}{180} R^{\mu \nu}(x) F\left(\square ; m^{2}\right) R_{\mu \nu}(x)\right. \\
\left.\left.+\frac{1}{2}\left(\frac{1}{6}-\xi\right)^{2} R(x) F\left(\square ; m^{2}\right) R(x)\right)+\mathcal{O}\left(m^{2} \mathcal{R}^{2}\right)\right]+\mathcal{O}\left(\mathcal{R}^{3}\right) .
\end{aligned}
$$


Here the $F\left(\square ; m^{2}\right)$ operator action should be understood through the expressions (44) and (45) as we did in the QED case (2); in addition we have neglected local finite terms and total derivatives. On the other hand, we see that the quadratic divergences agree with those obtained in the previous section.

Consider now the massless limit of the EA in (46). For small masses compared with $p$, the Mandelstam function behaves as shown in (6). As in the QED case, the term proportional to $\log \left(\mathrm{m}^{2}\right)$, associated with the divergences in (46), has the same coefficient but with the opposite sign to that coming from the Mandelstam function. Therefore, they exactly cancel. This allows us to obtain the regular massless limit:

$$
\begin{gathered}
W\left[g_{\mu \nu}\right]=\frac{1}{32 \pi^{2}} \int \mathrm{d} x \sqrt{g}\left(\frac{1}{180} R^{\mu \nu \lambda \rho}(x) \Gamma(\square) R_{\mu \nu \lambda \rho}(x)-\frac{1}{180} R^{\mu \nu}(x) \Gamma(\square) R_{\mu \nu}(x)\right. \\
\left.+\frac{1}{2}\left(\frac{1}{6}-\xi\right)^{2} R(x) \Gamma(\square) R(x)\right)+\mathcal{O}\left(\mathcal{R}^{3}\right)
\end{gathered}
$$

where $\Gamma(\square)$ is given in (8). This result is what one would expect on dimensional grounds [19], however, we have in addition an explicit representation, completely analogous to the flat spacetime case, for the form factors. In principle, the result in (46) cannot be considered, strictly speaking, as the $\mathcal{O}\left(\mathcal{R}^{2}\right)$ contribution in the curvature expansion since in the calculation we have not considered those terms with two curvatures and an arbitrary number of covariant derivatives, denoted by $\mathcal{O}\left(\nabla^{2} \mathcal{R}^{2}\right)$. We have obtained a non-local expression, but the infinite $\mathcal{O}\left(\nabla^{2} \mathcal{R}^{2}\right)$ terms could in principle modify it. However, we have seen in the previous section that the divergences have a logarithmic dependence on $\mathrm{m}^{2}$ (whose calculation is unambiguous). In addition, we showed in section 2 in the QED case that the coefficients of $\log \left(\mathrm{m}^{2}\right)$ in the divergent part have to be the same as those of $\log \left(p^{2}\right)$ in the non-local pieces, in order to have a regular massless limit. In (47) we have seen that our result does indeed possesses a regular massless limit. Since the $\mathcal{O}\left(\nabla^{2} \mathcal{R}^{2}\right)$ terms are finite, they could only modify the finite local and non-local part, but not the divergences. However, such a modification in the non-local part could spoil the regularity of the massless limit. Accordingly, we conclude that the $\mathcal{O}\left(\nabla^{2} \mathcal{R}^{2}\right)$ terms can only modify the local finite pieces and those proportional to $m^{2}$, but not the non-local ones containing $\log \left(\mathrm{m}^{2}\right)$. For that reason, the result in (46) includes all the quadratic curvature contributions except for those containing an arbitrary mass power denoted by $\mathcal{O}\left(m^{2} \mathcal{R}^{2}\right)$.

A compatible result has been obtained using partial resummation of the SchwingerDeWitt expansion [8] and also by means of the so-called covariant perturbation theory [9], in this case it was possible to derive the cubic terms in curvatures for asymptotically flat manifolds. Notice, however, that the boundary conditions that normal coordinates impose on the metric tensor are slightly more general than the asymptotically Minkowskian condition. In fact, we have only required that the curvatures and their covariant derivative vanish at infinity. This condition includes, for instance, those Robertson-Walker manifolds in which the expansion rate $\dot{a} / a$ asymptotically vanishes in the future, although the scale factor itself $a(t)$ does not tend to a constant. This kind of manifold is known to accept the definition of the so-called adiabatic vacua [13]. In [11] the possibility of defining such vacua was extremely useful for the interpretation of the effective action as the vacuum persistence amplitude.

To summarize, the procedure we have just presented makes possible a partial resummation of the higher-order terms in the EA, within the mentioned limits. Neglecting $\mathcal{O}\left(\mathcal{R}^{3}\right)$ terms implies that (46) will be a good approximation for $\nabla \nabla \mathcal{R} \gg \mathcal{R}^{2}$. In a similar fashion, when we neglect $\mathcal{O}\left(m^{2} \mathcal{R}^{2}\right)$ terms we are assuming that $\nabla \nabla \mathcal{R} \gg m^{2} \mathcal{R}$. However, if we are only interested in the two point Green functions with external curvature legs, the massless limit in (47) is exact. The renormalization of the EA can be done following the 
standard procedure in the classical references $[2,13]$. Here we will only mention that from the viewpoint of the Appelquist and Carrazone decoupling theorem [20], the scalar field does not decouple from gravity since there are new terms in the EA (46) which are not present in the Einstein-Hilbert action and they are not suppressed by powers of the particle mass $m$.

\section{Integration of the standard model matter fields: the EA for torsion}

Up to now we have only worked with scalar fields. However, in order to include the effect of the matter content present in the SM in the gravitational EA, we have to deal with the integration of fermionic fields. In this case we can proceed in a similar way using (14) in order to obtain the EA for the gauge fields and gravitation by integrating out the SM matter fields. The main novelty is that, in addition to the gravitational field (the vierbein), fermions also couple to the pseudotrace of the torsion as was discussed at the beginning of the previous section. The SM matter Lagrangian in a curved spacetime with torsion can be written as [21]

$$
\mathcal{L}_{M}=\sqrt{g}\left(\overline{\mathcal{Q}}\left(\mathrm{i} \not D^{Q}-M^{Q}\right) \mathcal{Q}+\overline{\mathcal{L}}\left(\mathrm{i} \not D^{L}-M^{L}\right) \mathcal{L}\right)
$$

where

$\not D^{Q}=\gamma^{\mu} D_{\mu}^{Q}=\gamma^{\mu}\left(\partial_{\mu}+\Omega_{\mu}^{Q}+S_{\mu}^{Q} \gamma_{5}\right), \quad \not D^{L}=\gamma^{\mu} D_{\mu}^{L}=\gamma^{\mu}\left(\partial_{\mu}+\Omega_{\mu}^{L}+S_{\mu}^{L} \gamma_{5}\right)$

with $M^{Q}$ and $M^{L}$ the mass matrices of quarks and leptons. We have followed the notation in [21]. In the following we will concentrate only on the gravitational couplings, so that we have neglected the gauge field contributions in the operators.

In order to obtain the torsion contribution to the EA up to $\mathcal{O}\left(S^{2}\right)$, we first consider a flat spacetime with torsion and afterwards we will include the effect of curvature. From the above Lagrangian we see that torsion behaves as the electromagnetic field in QED, without considering the $\gamma_{5}$ coupling, which does not affect the calculation of terms with an even number of fields, in the massless limit. Using the result in (2) and changing $e \not A \rightarrow-\frac{1}{8} \not S \gamma_{5}$, we obtain in this limit

$W[S]=-\left(\frac{1}{2} N_{\nu}+N_{D f}+N_{c} N_{q}\right) \frac{N_{f}}{192(4 \pi)^{2}} \int \mathrm{d}^{4} x S^{\mu v} \Gamma(\square) S_{\mu \nu}+\mathcal{O}\left(S^{4}\right)$

where $\Gamma(\square)$ is defined in (8), $N_{f}$ is the number of families, $N_{\nu}$ the number of neutrinos, $N_{D f}$ that of Dirac fermions, and $N_{q}$ the number of quark flavours. $S_{\mu \nu}=\partial_{\mu} S_{\nu}-\partial_{\nu} S_{\mu}$. Due to the absence of right neutrinos, there could be, in principle, parity-violating terms. However, it can be shown that those terms are total derivatives.

In order to introduce the spacetime curvature, we recall that the SM matter sector is locally conformally invariant (for massless fermions); this is also the case for the counterterms [22] (see $[2,23]$ where the renormalization procedure has been studied for manifolds with torsion). When including the curvature, apart from those in (50), there could be quadratic terms in torsion in the generic form $\mathcal{R} S^{2}$. However, such terms are not conformally invariant. Therefore, the ones obtained above are the only possible divergences. Next we will confirm these results with an explicit calculation of the divergences in a spacetime with curvature.

The SM Dirac operators in Euclidean space are not Hermitian because of the electroweak gauge couplings and the absence of the right neutrinos. However, in Euclidean space the EA divergences are real [18], thus it is enough to calculate $2 \operatorname{Re} W[e, \hat{\Gamma}]=-\log \operatorname{det}(\mathcal{O})$, where $\mathcal{O}=(\not D+M)^{\dagger}(\not D+M)$ and $\not D$ and $M$ denotes the joint Euclidean Dirac operator and the 
mass matrix for quarks and leptons. The heat-kernel expansion together with dimensional regularization $[13,24]$ allows us to obtain

$-\frac{1}{2} \operatorname{Tr} \log (\mathcal{O})=\frac{\mu^{\epsilon}}{2(4 \pi)^{D / 2}} \operatorname{tr} \sum_{n=0}^{\infty} M^{D-2 n} \Gamma\left(n-\frac{1}{2} D\right) \int \mathrm{d}^{4} x \sqrt{g} a_{n}(\mathcal{O}, x)$.

The well known heat-kernal coefficients $a_{n}$ are given for the above operators by

$$
\begin{gathered}
a_{0}(\mathcal{O}, x)=1, \quad a_{1}(\mathcal{O}, x)=\frac{1}{6} R-X \\
a_{2}(\mathcal{O}, x)=\frac{1}{12}\left[D_{\mu}, D_{\nu}\right]\left[D^{\mu}, D^{\nu}\right]+\frac{1}{6}\left[D_{\mu},\left[D^{\mu}, X\right]\right]+\frac{1}{2} X^{2}-\frac{1}{6} R X \\
-\frac{1}{30} R_{; \mu}{ }^{\mu}+\frac{1}{72} R^{2}+\frac{1}{180}\left(R_{\mu \nu \rho \sigma} R^{\mu \nu \rho \sigma}-R_{\mu \nu} R^{\mu \nu}\right)
\end{gathered}
$$

where, when we neglect the gauge fields contributions, the operator can be written as

$$
\mathcal{O}=D_{\mu} D^{\mu}+X+M^{2}
$$

with

$$
X=\gamma_{5} S_{; \mu}^{\mu}+2 S_{\mu} S^{\mu}-\frac{1}{4}\left[\gamma^{\mu}, \gamma^{\nu}\right]\left[d_{\mu}, d_{\nu}\right]
$$

and

$$
D_{\mu}=\partial_{\mu}+\Omega_{\mu}-\frac{1}{2} \gamma_{5}\left[\gamma_{\mu}, \gamma^{\nu}\right] S_{v}=d_{\mu}-\frac{1}{2} \gamma_{5}\left[\gamma_{\mu}, \gamma^{\nu}\right] S_{\nu}
$$

Writing the result in Lorentzian signature we have (see also the previous works [2, 23]):

$$
\begin{aligned}
W_{d i v}[e, \hat{\Gamma}]= & -\sum_{f} \sum_{i} N_{i} \frac{1}{32 \pi^{2}} \int \mathrm{d}^{4} x \sqrt{g}\left[M_{i}^{4}\left(\frac{1}{2} \Delta_{i}+\frac{3}{4}\right)-M_{i}^{2}\left(\Delta_{i}+1\right)\left(-\frac{1}{12} R+\frac{1}{32} S^{2}\right)\right. \\
& \left.+\Delta_{i}\left(\frac{1}{384} S^{\mu \nu} S_{\mu \nu}-\frac{7}{1440} R_{\mu \nu \rho \sigma} R^{\mu \nu \rho \sigma}-\frac{1}{180} R_{\mu \nu} R^{\mu \nu}+\frac{1}{288} R^{2}\right)\right]
\end{aligned}
$$

where $\sum_{f}$ denotes the sum over the different families and $\sum_{i}=\sum_{\text {leptons }}+N_{c} \sum_{\text {quarks }}$ over the different flavours in each family, including quarks and leptons. On the other hand, $N_{i}$ is the number of spinor components: 2 for neutrinos and 4 for the rest of the fermions. $M_{i}$ are the different fermion masses, $\Delta_{i}=N_{\epsilon}+\log \left(\mu^{2} / M_{i}^{2}\right)$ and $\mu$ the renormalization scale.

This formula is compatible with the flat spacetime result in (50). There is no $\mathcal{R} S^{2}$ term, as commented before, and we have discarded total derivatives. Following similar steps as for the scalar case, i.e. including $\log \left(\square / M^{2}\right)$ factors and taking the massless limit, we obtain the finite non-local contributions depending on curvature and torsion from the divergences. In the specified limit we have

$$
\begin{aligned}
& W[e, \hat{\Gamma}]=- \frac{N_{f}\left(8 N_{c}+6\right)}{32 \pi^{2}} \int \mathrm{d}^{4} x \sqrt{g}\left(\frac{1}{384} S^{\mu \nu} \Gamma(\square) S_{\mu \nu}+\frac{1}{288} R \Gamma(\square) R\right. \\
&\left.-\frac{7}{1440} R_{\mu \nu \rho \sigma} \Gamma(\square) R^{\mu \nu \rho \sigma}-\frac{1}{180} R_{\mu \nu} \Gamma(\square) R^{\mu \nu}\right)+\mathcal{O}\left(\mathcal{R}^{3}\right)+\mathcal{O}\left(S^{3}\right)
\end{aligned}
$$

where $\Gamma(\square)$ is given in $(8)$ and $\mathcal{O}\left(S^{3}\right)$ denotes terms with three or more torsion fields. Finally, due to the presence of chiral fermions, the EA could contain an abnormal parity sector, responsible for the gauge and gravitational anomalies. However, as the previous result has been derived from the real part of the EA (with normal parity), this sector is not taken into account.

The renormalization procedure in this case will require, not only the introduction of quadratic terms and a constant, but also a kinetic and mass terms for the torsion field. Accordingly, the starting classical action should be

$S_{G}=\int \mathrm{d} x \sqrt{g}\left(\frac{R-2 \Lambda}{16 \pi G}+a_{2} R^{\mu \nu \lambda \rho} R_{\mu \nu \lambda \rho}+a_{3} R^{\mu \nu} R_{\mu \nu}+a_{4} R^{2}+a_{5} S_{\mu \nu} S^{\mu \nu}+a_{6} S^{2}\right)$. 
Table 1. Renormalization constants for the SM case.

\begin{tabular}{llll}
\hline$C_{0}$ & $C_{1}$ & $C_{2}$ & $C_{3}$ \\
\hline$-\sum_{f} \sum_{i} N_{i} M_{i}^{4} / 2$ & $-\sum_{f} \sum_{i} N_{i} M_{i}^{2} / 12$ & $\frac{7}{180} N_{f} N_{c}+\frac{7}{240} N_{f}$ & $\frac{2}{45} N_{f} N_{c}+\frac{1}{30} N_{f}$ \\
\hline$C_{4}$ & $C_{5}$ & $C_{6}$ \\
\hline$-\frac{1}{36} N_{f} N_{c}-\frac{1}{48} N_{f}$ & $-\frac{1}{48} N_{f} N_{c}-\frac{1}{64} N_{f}$ & $\sum_{f} \sum_{i} N_{i} M_{i}^{2} / 32$ & \\
\hline
\end{tabular}

The divergences can be absorbed by a redefinition of the constants:

$$
a_{j} \rightarrow a_{j}^{\prime}=a_{j}^{r}(\mu)-\frac{1}{32 \pi^{2}} C_{j} N_{\epsilon}+\text { finite constants, } \quad j=0, \ldots, 6
$$

where $a_{0}=-2 \Lambda /(16 \pi G)$ and $a_{1}=1 /(16 \pi G)$. The values of $C_{j}$ are shown in table 1 .

The scale dependence of the renormalized constants is derived from the renormalization group equation [2,23], in an analogous way to the scalar case. Consequently, if at a given scale $\mu$, the constants have certain values $a_{i}^{r}(\mu)$, their values at a different scale $\mu^{\prime}$ will be given by

$$
a_{i}^{r}\left(\mu^{\prime}\right)=a_{i}^{r}(\mu)+\frac{C_{i}}{32 \pi^{2}} \log \left(\frac{\mu^{2}}{\mu^{\prime 2}}\right)
$$

where the $C_{i}$ constants are those appearing in table 1 . In particular, the renormalized Newton constant $G^{r}(\mu)$ has a scale dependence and therefore its value should be specified for a given $\mu$. Thus $G$ will depend on the size of the system we are considering and this could have enormous importance in cosmology (see [25]).

In the renormalized EA we can generate a torsion kinetic term by means of an $S_{v}$ finite normalization as follows: $S_{v}^{r}=Z_{3}^{-1 / 2}(\mu) S_{v}$, where $a_{5}^{r}(\mu)=-\frac{1}{4} Z_{3}^{-1}(\mu)$. Thus in the massless limit we have

$$
W^{r}\left[S^{r}\right]=\int \mathrm{d}^{4} x \sqrt{g}\left(-\frac{1}{4} S_{\mu \nu}^{r} S^{r \mu \nu}+\text { non-local terms }+\mathcal{O}\left(S^{3}\right)\right) .
$$

Consequently, the physical torsion field $S_{\mu}^{r}$ will behave as an Abelian gauge field. Therefore, we have generated a kinetic term for torsion even when starting from a theory without propagating torsion.

\section{Conclusions and discussion}

In this work we have dealt with the computation of the low-energy effective action for gravity obtained when matter fields, both scalar and fermionic, are integrated out. As a much simpler exercise, we have started by reviewing the low-energy EA for the electromagnetic field obtained when the electronic field is integrated out. Special attention has been paid to the non-local terms which are related to the particle production probabilities.

In order to integrate the scalar fields to compute the EA for gravity, we have used the normal coordinate expansion. This method has been used previously to compute the divergent terms of the EA. By comparison of this kind of expansion around different spacetime points, we have been able to extend the previous work to also obtain the non-local finite terms of the EA up to quadratic terms in the curvature. In particular, we have arrived at the conclusion that the scalar field does not decouple from the EA in the large-mass limit in the AppelquistCarrazone sense. Finally, we have also discussed the meaning of the expansion in the massless limit. 
In order to be able to consider the matter field content present in the standard model we have also studied the case of fermionic matter. The main novelty in this case is that this kind of field can also couple to the torsion pseudotrace in addition to the gravitational field. Thus we have obtained the low-energy EA for the gravitational field and the torsion including the divergent and the non-local finite terms up to quadratic terms in the curvature and the torsion. We have discussed the renormalization of this EA and we have found that a kinetic term is generated for the torsion pseudotrace. This result is quite interesting since in the standard Einstein-Cartan action [1], the torsion does not propagate.

Finally, we would like to stress that the results obtained in this work can be useful for the study of some interesting physical effects. In particular, the low-energy EA can be applied for the study of the quantum stability of classical solutions of the Einstein equations of motion. More specifically, the non-local terms of the EA which have been computed here, are complex in general. Classical solutions that give rise to an imaginary part when the EA is evaluated on them, are unstable by particle radiation even if they are stable at the classical level. Moreover, the imaginary part of the EA evaluated on classical solutions, can be used to compute particle production rates and spectra, thus providing an alternative method to the more commonly used one based on the Bogolyubov transformations. The expression that we have obtained for the non-local form factors is particularly appropriate for the calculation of particle production probabilities in cosmological spacetimes. The boundary conditions imposed by the normal coordinates expansion, allow one to apply this method not only to asymptotically flat manifolds, but also to some other cosmological manifolds where the expansion rate vanishes asymptotically. Work has been done in this direction and it will be presented elsewhere [11].

\section{Acknowledgments}

We thank I L Shapiro for his comments and suggestions on several points of the paper. This work has been partially supported by the Ministerio de Educación y Ciencia (Spain) (CICYT AEN96-1634). ALM acknowledges support from SEUID-Royal Society.

\section{Appendix}

\section{Dimensional regularization formulae}

In dimensional regularization [26], the spacetime dimension is taken to be $D=4-\epsilon$ and the poles are parametrized through $N_{\epsilon}$ which was defined in section 2 . We will use the notation $\mathrm{d} \tilde{q}=\mu^{\epsilon} \mathrm{d}^{D} q /(2 \pi)^{D}$ where $\mu$ is the renormalization scale. Some useful expressions are:

$$
\begin{gathered}
\int \mathrm{d} \tilde{q} \frac{\left(q^{2}\right)^{r}}{\left[q^{2}-R^{2}\right]^{m}}=\mathrm{i} \frac{(-1)^{r-m}}{(4 \pi)^{D / 2}} \frac{\Gamma(r+D / 2) \Gamma(m-r-D / 2) \mu^{\epsilon}}{\Gamma(D / 2) \Gamma(m)\left(R^{2}\right)^{m-r-D / 2}} \\
I_{1}\left(p, m^{2}\right) \equiv \int \mathrm{d} \tilde{q} \frac{1}{\left(q^{2}-m^{2}\right)^{2}\left((q+p)^{2}-m^{2}\right)} \\
=\mathrm{i} \frac{(-1)^{D / 2}}{(4 \pi)^{D / 2}} \Gamma(3-D / 2) \int_{0}^{1} \mathrm{~d} t(1-t)\left(-m^{2}+p^{2} t(1-t)\right)^{D / 2-3} \\
\int^{m^{2}} I_{1}\left(p, m^{2}\right) \mathrm{d} m^{2}=\frac{i \mu^{\epsilon}}{2(4 \pi)^{2}}\left(N_{\epsilon}+2-\log \frac{m^{2}}{\mu^{2}}-F\left(p^{2} ; m^{2}\right)\right)
\end{gathered}
$$




$$
\begin{aligned}
I_{2}^{\mu \nu}\left(p, m^{2}\right) \equiv & \int \mathrm{d} \tilde{q} \frac{q^{\mu} q^{\nu}}{\left(q^{2}-m^{2}\right)^{3}\left((q+p)^{2}-m^{2}\right)} \\
= & \mathrm{i} \frac{(-1)^{D / 2}}{2(4 \pi)^{D / 2}} \Gamma(4-D / 2) \int_{0}^{1} \mathrm{~d} t(1-t)^{2}\left(-m^{2}+p^{2} t(1-t)\right)^{D / 2-4} \\
& \times\left(p^{\mu} p^{\nu}+g^{\mu \nu} \frac{-m^{2}+p^{2} t(1-t)}{2(3-D / 2)}\right) \\
\int_{3}^{m^{2}} I_{2}^{\mu \nu}\left(p, m^{2}\right) & \mathrm{d} m^{2}=\frac{i \mu^{\epsilon}}{12(4 \pi)^{2}}\left(N_{\epsilon}+\frac{13}{6}-\log \frac{m^{2}}{\mu^{2}}-F\left(p^{2} ; m^{2}\right)\right) g^{\mu \nu}+\mathcal{O}\left(p^{2}\right) \\
I_{3}^{\mu \nu \alpha \beta}\left(p, m^{2}\right) \equiv & \int \mathrm{d} \tilde{q} \frac{q^{\mu} q^{v} q^{\alpha} q^{\beta}}{\left(q^{2}-m^{2}\right)^{4}\left((q+p)^{2}-m^{2}\right)} \\
= & \mathrm{i} \frac{(-1)^{D / 2}}{6(4 \pi)^{D / 2}} \Gamma(5-D / 2) \int_{0}^{1} \mathrm{~d} t(1-t)^{3}\left(-m^{2}+p^{2} t(1-t)\right)^{D / 2-3} \\
& \times \frac{g^{\alpha \nu} g^{\beta \mu}+g^{\alpha \mu} g^{\beta \nu}+g^{\alpha \beta} g^{\mu \nu}}{(6-D)(8-D)}+\mathcal{O}\left(p^{2}\right) \\
\int_{3}^{m^{2}} I_{3}^{\mu \nu \alpha \beta}(p, & \left.m^{2}\right) \mathrm{d} m^{2}=\frac{\mathrm{i} \mu^{\epsilon}}{96(4 \pi)^{2}}\left(N_{\epsilon}+\frac{7}{3}-\log \frac{m^{2}}{\mu^{2}}-\left(1-\frac{2 m^{2}}{p^{2}}\right) F\left(p^{2} ; m^{2}\right)-\frac{4 m^{2}}{p^{2}}\right) \\
& \times\left(g^{\alpha \nu} g^{\beta \mu}+g^{\alpha \mu} g^{\beta \nu}+g^{\alpha \beta} g^{\mu \nu}\right)+\mathcal{O}\left(p^{2}\right)
\end{aligned}
$$

where $F\left(p^{2} ; M^{2}\right)$ is given in (4). These expressions have been obtained in the Minkowski spacetime. We have not explicitly included the $+\mathrm{i} \epsilon$ terms accompanying the momenta in order to avoid the confusion with terms coming from dimensional regularization.

\section{Normal coordinates expansions}

We will show the Riemann normal coordinate expansions for different objects up to order $\mathcal{O}\left(\partial^{4}\right)$. We will take the point $y_{0}$ as the coordinates origin. For the inverse metric tensor we have

$$
\begin{aligned}
& g^{\mu \nu}(y)=\eta^{\mu \nu}-\frac{1}{3} R_{\alpha \beta}^{\mu}{ }_{\alpha}^{\nu}\left(y_{0}\right) y^{\alpha} y^{\beta}+\frac{1}{6} R_{\alpha \beta ; \gamma}^{\mu \nu}\left(y_{0}\right) y^{\alpha} y^{\beta} y^{\gamma} \\
& +\left[-\frac{1}{20} R_{\alpha \beta ; \gamma \delta}^{\mu}\left(y_{0}\right)+\frac{1}{15} R_{\alpha}{ }^{\mu}{ }_{\beta \lambda}\left(y_{0}\right) R_{\gamma}^{\lambda}{ }^{v}{ }_{\delta}\left(y_{0}\right)\right] y^{\alpha} y^{\beta} y^{\gamma} y^{\delta}+\mathcal{O}\left(\partial^{5}\right) .
\end{aligned}
$$

The metric determinant $g=\left|\operatorname{det} g_{\mu \nu}\right|$ has the following expansion:

$$
\begin{aligned}
g(y)=1+\frac{1}{3} & R_{\alpha \beta}\left(y_{0}\right) y^{\alpha} y^{\beta}-\frac{1}{6} R_{\alpha \beta ; \gamma} y^{\alpha} y^{\beta} y^{\gamma} \\
& +\left(\frac{1}{18} R_{\alpha \beta} R_{\gamma \delta}-\frac{1}{90} R_{\lambda \alpha \beta \kappa} R^{\lambda}{ }_{\gamma}{ }^{\kappa}{ }_{\delta}+\frac{1}{20} R_{\alpha \beta ; \gamma \delta}\right) y^{\alpha} y^{\beta} y^{\gamma} y^{\delta}+\mathcal{O}\left(\partial^{5}\right) .
\end{aligned}
$$

For the square root of the metric determinant and its inverse we have

$$
\begin{aligned}
g^{1 / 2}(y)=1+ & \frac{1}{6} R_{\alpha \beta}\left(y_{0}\right) y^{\alpha} y^{\beta}-\frac{1}{12} R_{\alpha \beta ; \gamma} y^{\alpha} y^{\beta} y^{\gamma} \\
& +\left(\frac{1}{72} R_{\alpha \beta} R_{\gamma \delta}-\frac{1}{180} R_{\lambda \alpha \beta \kappa} R^{\lambda}{ }_{\gamma}{ }^{\kappa}{ }_{\delta}+\frac{1}{40} R_{\alpha \beta ; \gamma \delta}\right) y^{\alpha} y^{\beta} y^{\gamma} y^{\delta}+\mathcal{O}\left(\partial^{5}\right), \\
g^{-1 / 2}(y)=1- & \frac{1}{6} R_{\alpha \beta}\left(y_{0}\right) y^{\alpha} y^{\beta}+\frac{1}{12} R_{\alpha \beta ; \gamma} y^{\alpha} y^{\beta} y^{\gamma} \\
& +\left(\frac{1}{72} R_{\alpha \beta} R_{\gamma \delta}+\frac{1}{180} R_{\lambda \alpha \beta \kappa} R^{\lambda}{ }_{\gamma}{ }^{\kappa}{ }_{\delta}-\frac{1}{40} R_{\alpha \beta ; \gamma \delta}\right) y^{\alpha} y^{\beta} y^{\gamma} y^{\delta}+\mathcal{O}\left(\partial^{5}\right) .
\end{aligned}
$$




\section{References}

[1] Hehl F W, Heyde P, Kerlick G D and Nester J M 1976 Rev. Mod. Phys. 48393

[2] Buchbinder IL, Odintsov S D and Shapiro I L 1992 Effective Action in Quantum Gravity (Bristol: IOP Publishing)

[3] 't Hooft G and Veltman M 1974 Ann. Inst. H Poincaré 20245

[4] Capper D M, Leibrandt G and Ramón Medrano M 1973 Phys. Rev. D 84320

Brown M R 1973 Nucl. Phys. 56194

Capper D M, Duff M J and Halpern L 1974 Phys. Rev. D 10461

Capper D M and Duff M J 1974 Nucl. Phys. B 82147

[5] Deser S and van Niewenhuizen P 1974 Phys. Rev. Lett. 32245

Deser S and van Niewenhuizen P 1974 Phys. Rev. D 10401

Deser S and van Niewenhuizen P 197410411

Deser S, Tsao H S and van Niewenhuizen P 1974 Phys. Rev. D 103337

[6] Schwinger J 1951 Phys. Rev. 82664

[7] DeWitt B S 1965 Dynamical Theory of Groups and Fields (New York: Gordon and Breach)

[8] Avramidi I G 1991 Nucl. Phys. B 355712

[9] Barvinsky A O and Vilkovisky G A 1987 Nucl. Phys. B 282163

Barvinsky A O and Vilkovisky G A 1990 Nucl. Phys. B 333471

Barvinsky A O and Vilkovisky G A 1990 Nucl. Phys. B 333512

Barvinsky A O, Gusev Yu V, Vilkovisky G A and Zhytnikov V V 1995 Nucl. Phys. B 439561

[10] Bunch T S and Parker L 1979 Phys. Rev. D 202499

[11] Dobado A and Maroto A L 1999 Phys. Rev. D to appear

(Dobado A and Maroto A L 1998 Preprint gr-qc/9803076)

[12] Heisenberg W and Euler H 1936 Z. Phys. 98714

Dobado A, Gómez-Nicola A, Maroto A L and Peláez J R 1997 Effective Lagrangians for the Standard Model (Berlin: Springer)

[13] Birrell N D and Davies P C W 1982 Quantum Fields in Curved Space (Cambridge: Cambridge University Press)

[14] Petrov A Z 1969 Einstein Spaces (Oxford: Pergamon)

[15] Eisenhart L P 1964 Riemannian Geometry (Princeton, NJ: Princeton University Press)

[16] Cognola G and Zerbini S 1987 Phys. Lett. B 195435

[17] Vainshtein A I, Zakharov V I, Novikov V A and Shifman M A 1984 Sov. J. Nucl. Phys. 3977 Zuk J A 1985 Phys. Rev. D 322653

[18] Ball R D 1989 Phys. Rep 1821

[19] Deser S, Duff M J and Isham C J 1976 Nucl. Phys. B 11145

[20] Appelquist T and Carazzone J 1975 Phys. Rev. D 112856

[21] Dobado A and Maroto A L 1996 Phys. Rev. D 545185

[22] Duff M J 1977 Nucl. Phys. B 215334

[23] Buchbinder I L and Shapiro I L 1985 Phys. Lett. B 151263

Buchbinder I L and Shapiro I L 1990 Class. Quantum Grav. 71197

Buchbinder I L, Odintsov S D and Shapiro I L 1985 Phys. Lett. B 16292

[24] Jack I and Osborn H 1984 Nucl. Phys. B 234331

[25] Goldman T, Pérez-Mercader J, Cooper F and Martín-Nieto M 1992 Phys. Lett. B 281219

[26] 't Hooft G and Veltman M 1972 Nucl. Phys. B 44189

't Hooft G and Veltman M 1979 Nucl. Phys. B 153365

Pascual P and Tarrach R 1984 QCD: Renormalization for the Practitioner (Berlin: Springer) 